\title{
Application of digital cholangioscopy assisted non- radiation endoscopic retrieval of common bile duct stones
}

\section{Yadong Feng ( $\sim$ drfengyd@126.com )}

Southeast University Zhongda Hospital https://orcid.org/0000-0001-9259-3840

\section{Wei Xu}

Southeast University Zhongda Hospital

\section{Yang Liu}

Southeast University Zhongda Hospital

\section{Xiaomei Sun}

Southeast University Zhongda Hospital

\section{Yan Liang}

Southeast University Zhongda Hospital

\section{Chibin Bu}

Southeast University Zhongda Hospital

\section{Yuanyuan Li}

Southeast University Zhongda Hospital

\section{Youyu Zhang}

Southeast University Zhongda Hospital

\section{Jiong Zhang}

Southeast University Zhongda Hospital

\section{Ruihua Shi}

Southeast University Zhongda Hospital

\section{Technical advance}

Keywords: digital cholangioscopy, non-radiation ERCP, choledocholithiasis, endoscopic therapy

Posted Date: July 24th, 2020

DOI: https://doi.org/10.21203/rs.3.rs-41631/v1

License: (c) (i) This work is licensed under a Creative Commons Attribution 4.0 International License.

Read Full License 


\section{Abstract}

Background Few studies have evaluated digital cholangioscopy (DCS) assisted non-radiation endoscopic retrograde cholangiopancreatography (NR-ERCP) for choledocholithiasis. Here, we evaluated the application of DCS assisted NR-ERCP for endoscopic retrieval of common bile duct (CBD) stones.

Methods Patients who underwent ERCP for choledocholithiasis and without prior ERCP were included. Data related to technical success and outcomes of DCS-assisted NR-ERCP and conventional ERCP were retrieved and compared. Procedure and technical details of DCS assisted NR-ERCP were collated and reviewed.

Results In total, 304 and 53 patients who underwent conventional and DCS-assisted ERCP were recruited. Relatively larger stones $(p<0.001)$ was present in DCS assisted NR-ERCP. No statistical difference was present in biliary access, lithotripsy, stone removal and total complications. A higher proportion of larger balloon for EPBD $(p<0.001)$ and more ERCP sessions for stone clearance $(p<0.001)$ were present in conventional ERCP. In DCS-assisted ERCP, technical success was achieved in 52 (98.1\%) patients and DCS-guided laser lithotripsy was applied in $9(17 \%)$ patients. DCS guided laser lithotripsy was superior to mechanical lithotripsy for stone clearance $(p<0.001)$ in large stones. Mean length of biliary exploration and whole NR-ERCP were $8.60 \pm 1.96(6-19)$ and $32.96 \pm 16.29$ (13-82) minutes, respectively. One delayed bile-leakage, one moderate pancreatitis, two mild pancreatitis and five cases of hyperamylasemia occurred. Technical details and complications were not statistically differed between patients with a dilated and a non-dilated CBD.

Conclusions DCS-based NR-ERCP is technically feasible and safe for retrieval of CBD stones. It provides an alternative to conventional ERCP for endoscopic treatment of simple choledocholithiasis.

\section{Background}

Endoscopic retrograde cholangiopancreatography (ERCP) is an established therapeutic method for removal of common bile duct (CBD) stones [1]. Fluoroscopy-aided ERCP remains the standard and predominant modality worldwidely. Despite of application of low-dose radiation X-ray system in ERCP, the use of fluoroscopy places patients and endoscopists at risk of radiation-induced injury [2]. Although radiation time and dosage can be minimized by experienced ERCP endoscopists [3], how to reduce radiation exposure during conventional ERCP procedures has gained great attention [4]. According to the ALARA (As Low as Reasonably Achievable) principle, precautions should be taken to reduce radiation exposure to patients and staff. On one hand, some studies have discussed how to deal with cumulative radiation exposure to endoscopists during ERCP [5]. On the other hand, radiation protection for ERCP in particular patients, such as pregnant women and children, has been emphasized [6].

Using a non-radiation strategy may be an alternative for controlling and minimizing in-procedure radiation. To avoid potential radiation caused health consequences, there has been interest in the use of non-radiation ERCP (NR-ERCP) in recent years [7-10]. There are two major techniques of NR-ERCP, 
namely endoscopic ultrasound assisted [7, 8] and digital cholangioscopy (DCS)-assisted [9, 10] ERCP. Recently, a commercially available DCS (SpyGlass DS ${ }^{\mathrm{TM}}$, Boston Scientific, Marlborough, MA, USA), which can provide a good visualization of the biliary tree, makes the NR-ERCP as a much easier process [9-11]. Furthermore, DCS seems to be more user-friendly and conveniently manipulated [11]. However, DCSassisted NR-ERCP is limited performed. Subsequently, DCS-assisted NR-ERCP remains to be discussed. In this study, we present our experience of DCS-assisted NR-ERCP for retrieval of common bile duct stones.

\section{Methods}

\section{Study design and patients enrollment}

DCS-assisted NR-ERCP is available since July 2019 in our center. Due to a high cost of DCS, an inducible recommendation of DCS was strictly forbidden. Patients who underwent conventional ERCP were set as the control group. Data on technical details and outcomes of two modalities of ERCP were compared. Procedure details and technical details of DCS-assisted NR-ERCP were reviewed in detail.

Patients underwent ERCP for choledocholithiasis were recruited in this study. The exclusion criteria were as follows: (1) Billroth II or Roux-en-Y anatomy; (2) coagulopathy (platelet count $<50,000 / \mu \mathrm{L}$ or prothrombin time international normalized ratio > 2.0); (3) pregnancy; (4) moderate and severe acute cholangitis [12]; (5) obvious biliary stricture distal to stones; (6) suspicion of biliary malignancy; (7) prior history of endoscopic sphincterotomy (EST); (8) refusal to undergo ERCP. Informed consent was obtained from all patients before ERCP. All ERCPs were performed under general anesthesia.

\section{Brief procedures of two modalities of ERCP}

In NR-ERCP, patients were placed in a left lateral decubitus position. As showed in Fig. 1, key steps of NRERCP were followings. (1) Standard guidewire-assisted biliary cannulaution using a sphincterotome (Microtech, Nanjing, China) and a 0.035-inch guidewire (Microtech, Nanjing, China), which is confirmed by visible bile aspiration. If the pancreatic duct was cannulated more than five times, a pancreatic stent was inserted. Advanced cannulation techniques were applied if necessary. (2) A small EST combined with endoscopic papillary balloon dilation (EPBD) was performed. The size of the balloon was determined according to the diameter of the distal CBD by a latest imaging. (3) A DCS biliary exploration, from the papilla to the hilum, was performed. (4) After biliary exploration, a basket or balloon was used for stones extraction. Laser lithotripsy was applied for large stones. (5) A second round DCS biliary exploration was performed to confirm clearance of stones. If there was residual stone, an additional stone extraction was performed. (6) A 7F double pig-tail stent was placed for temporary biliary drainage, and was removed before discharge.

Conventional ERCP was routinely performed. Briefly, biliary cannulation, cholangiography, small EST and EPBD and a basket or balloon extraction of CBD stones were sequentially performed. Mechanical lithotripsy was applied if necessary. Clearance of CBD stones was confirmed by a cholangiography. After 
clearance of stones, a temporary endoscopic nasal biliary drainage was performed and was removed before discharge. A biliary stenting was performed in case of difficult large stones or stones residual.

\section{Post-procedure evaluation}

All patients received clinical observation after ERCP. Post-ERCP complications were defined by Cotton's criteria [13]. Anesthesia-related complications were defined as a previous study by Rex et al [14].

\section{Statistics}

Quantitative data are presented as mean \pm standard deviation (SD) while categorical data are expressed as frequencies and percentages. The Mann-Whitney $U$ test, Chi-square test and one-way analysis of variance (ANOVA) were performed using SPSS for Windows, version 20.0 (SPSS Inc., Chicago, IL, USA). A $p$ value $<0.05$ was considered to be statistically significant.

\section{Results}

\section{Patients and basal characteristics}

Based on our database, 387 patients underwent conventional ERCP for choledocholithiasis in our center between January 2017 and February 2020. Among these, 304 patients were without a prior ERCP. From July 2019 to February 2020, 56 consecutive patients with CBD stones underwent DCS-assisted NR-ERCP. Three patients who had a prior EST were excluded. Subsequently, 357 patients with a naive papilla were retrospectively included. Patient characteristics were listed in Table 1. There was no statistical difference regarding age $(p=0.633)$, gender $(p=0.766)$, numbers of CBD stones $(p=0.102)$ and presence of a periampullary diverticulum (PAD, $p=0.192)$. Although mean size of CBD stones was not statistically differed $(p=0.235)$, relatively lager stones were included in DCS group $(p<0.001)$. 
Table 1

Characteristics of enrolled patients

\begin{tabular}{|llll|}
\hline Patient's characteristics & $\begin{array}{l}\text { C-ERCP } \\
(\mathbf{n}=304)\end{array}$ & $\begin{array}{l}\text { D-ERCP } \\
(\mathbf{n}=53)\end{array}$ & p-value \\
\hline Mean age, years(range) & $65.60 \pm 15.93$ & $64.45 \pm 14.53$ & 0.633 \\
& $(19-89)$ & $(18-90)$ & \\
Gender, $n(\%)$ & $157(51.6)$ & $29(54.7 \%)$ & 0.766 \\
Male & $147(48.4)$ & $24(45.3)$ & \\
Female & & & 0.102 \\
Stone details & $7(2.3)$ & $4(7.5)$ & 0.235 \\
Stone numbers, $n(\%)$ & $242(79.6)$ & $38(7.17)$ & 0.001 \\
Suspection & $55(18.1)$ & $11(20.8)$ & \\
$1-3$ & $11.50 \pm 2.94$ & $10.92 \pm 4.82$ & \\
$\geq 4$ & $(0-20)$ & $(0-26)$ & \\
Mean size of stones, mm(range) & $53(17.4)$ & $15(28.3)$ & \\
Numbers of stone in size, $n(\%)$ & $226(74.3)$ & $30(56.6)$ & \\
$0-9$ mm & $25(8.2)$ & $6(11.3)$ & \\
$10-15$ mm & $0(0)$ & $2(3.8)$ & \\
$16-20$ mm & & & \\
$20-26$ mm & $86(28.3)$ & $20(37.7)$ & \\
\hline PAD & & & \\
\hline C-ERCP: conventional ERCP; D-ERCP: DCS-assisted ERCP; PAD: periampullary diverticulum & \\
\hline
\end{tabular}

\section{Comparison of technical details and outcomes between two modalities of ERCP}

These results were listed in Table 2. There was no statistical difference in respect of successful biliary access $(p=0.331)$, application of lithotripsy $(p=0.093)$ and total complications $(p=0.630)$. Although total stone removal $(p=0.17)$ was not statistically differed, a higher proportion of larger balloon for EPBD $(p<0.001)$ and more ERCP sessions for stone clearance $(p<0.001)$ were present in conventional ERCPs. For retrieval of large stones, DCS-guided laser lithotripsy was superior to mechanical lithotripsy by presenting a higher stone clearance $(100 \%$ vs. $70.6 \%, p<0.001)$. 
Table 2

Comparison of technical details and outcomes of two modalities of ERCP

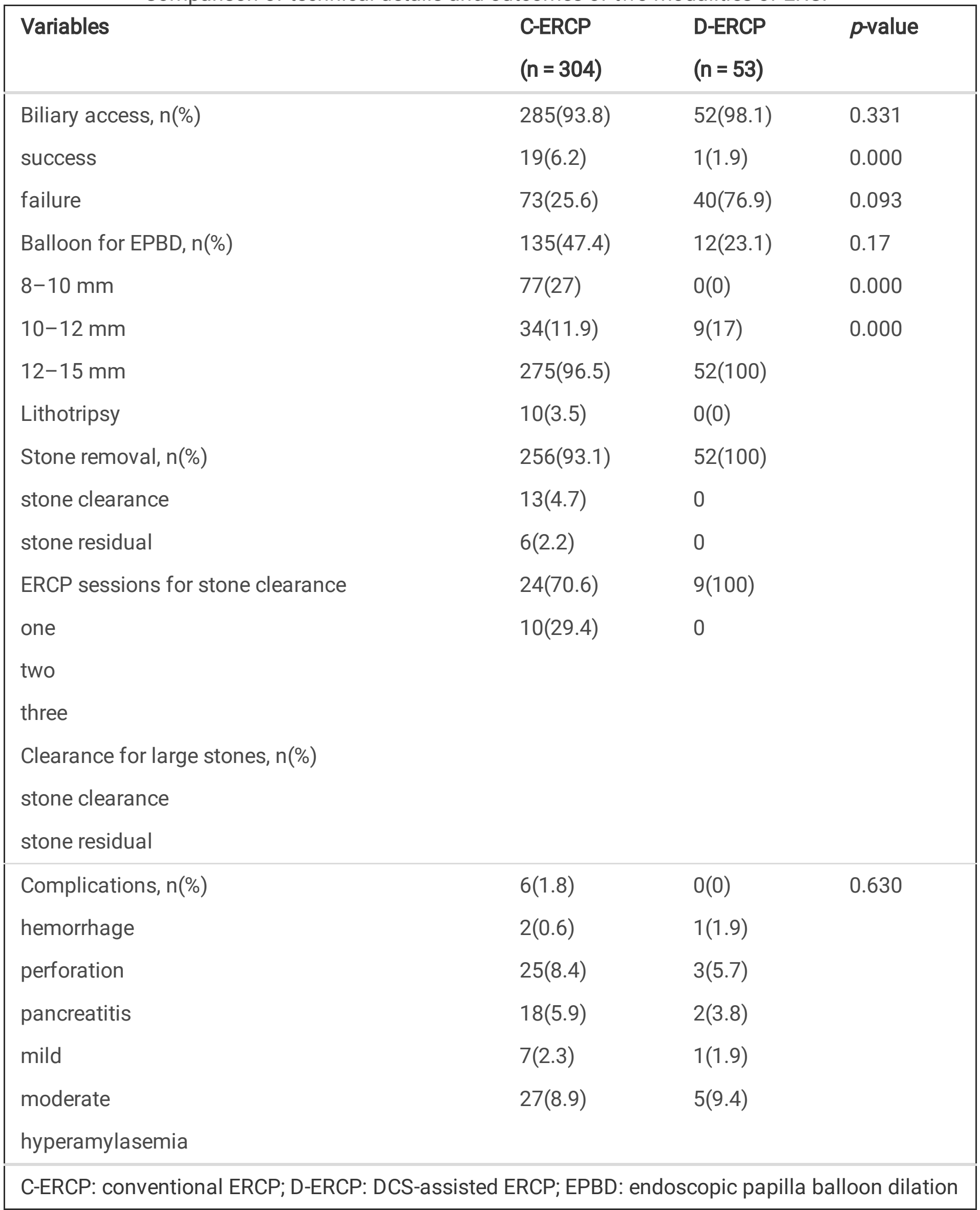


As showed in Table 3, standard cannulation, double-guidewire technique and transpancreatic pre-cut were applied in 48, 2 and 2 patients, respectively. The mean time of successful biliary cannualtion was $56.13 \pm$ 91.15 (9-518) seconds. Non-radiation biliary cannulation failed in another patient; and biliary access was not achieved by fluoroscopy guidance.

Table 3

Endoscopic details of NR-ERCP

\begin{tabular}{|ll|}
\hline NR-ERCP details & \\
\hline Biliary cannulation, $\mathrm{n}(\%)$ & $49(100)$ \\
standard technique & $45(91.8)$ \\
advanced techniques & $4(8.2)$ \\
double guidewire technique & $2(4.1)$ \\
pre-cut technique & $2(4.1)$ \\
\hline CBD stones removal & $2(0-9)$ \\
stone numbers, mean(range) & $48(92.3)$ \\
discrete stones, $\mathrm{n}(\%)$ & $4(7.7)$ \\
stone sludge, $\mathrm{n}(\%)$ & $9(17)$ \\
laser lithotripsy, $\mathrm{n}(\%)$ & \\
\hline Procedure time, minutes (range) & $8.60 \pm 1.96(6-19)$ \\
biliary exploration & $32.96 \pm 16.29(13-82)$ \\
whole NR-ERCP & \\
\hline NR-ERCP: non-radiation ERCP; CBD: common bile duct
\end{tabular}

Discrete stones and stone sludge were visualized in 46 (86.8\%) and 6 (11.5\%) patients. Laser lithotripsy was applied in $9(17 \%)$ patients. In one female patient, multiple stones were present by magnetic resonance cholangiopancreatography; however, cracked stones were found by DCS biliary exploration (Fig. 2). Consequently, a basket stone extraction was performed instead of planned laser lithotripsy. The mean time of biliary exploration and whole NR-ERCP were $8.60 \pm 1.96(6-19)$ and $32.96 \pm 16.29(13-82)$ minutes, respectively.

Setting $10 \mathrm{~mm}$ as a cut-off value, 27 and 26 patients were with a dilated and a non-dilated CBD. There was no difference in regarding of whole procedure time (31.23 \pm 18.94 vs. $34.63 \pm 13.42$ minutes, $p=$ $0.453)$, cannulation techniques $(p=0.199)$, duration of biliary exploration $(8.36 \pm 1.95$ vs. $8.81 \pm 1.98$ minutes, $p=0.409)$, guidewire-assisted DCS advancement $(p=0.522)$ and complications $(p=0.287)$. These results of stratified analysis were listed in Table 4. 
Table 4

Stratified analysis of technical details and adverse events in patients with a dilated and non-dilated CBD

Diameter of CBD

value

\begin{tabular}{|llll|} 
& $\begin{array}{l}\text { Dilated } \\
10 \mathrm{~mm}) \\
(\mathbf{n = 2 7})\end{array}$ & $\begin{array}{l}\text { Non-dilated }(< \\
10 \mathrm{~mm}) \\
(\mathbf{n = 2 6})\end{array}$ & 0.453 \\
\hline $\begin{array}{l}\text { Whole ERCP time, minutes } \\
\text { (mean } \pm \text { SD) }\end{array}$ & $31.23 \pm 18.94$ & $34.63 \pm 13.42$ & 0.199 \\
\hline $\begin{array}{l}\text { Biliary cannulation } \\
\text { Standard technique }\end{array}$ & 24 & 23 & \\
Double guidewire techiniqe & 1 & 2 & 0.409 \\
Pre-cut & 2 & 0 & 0.522 \\
\hline $\begin{array}{l}\text { Duration of biliary exploration, minutes (mean } \\
\pm \text { SD) }\end{array}$ & $8.36 \pm 1.95$ & $8.81 \pm 1.98$ & 0.287 \\
\hline Guidewire assisted DCS advancement & 5 & 4 & \\
\hline Adverse events & 0 & 1 & \\
perforation & 0 & 3 & \\
pancreatitis & 2 & 3 & \\
\hline hyperamylasemia & & & \\
\hline CBD: common bile duct & & & \\
\hline
\end{tabular}

Complications were listed in Table 2. One delayed perforation occurred in one male patient with a large type III PAD and a non-dilated CBD. He underwent a small EST followed by a 10-mm EPBD, and suffered from a delayed bile-leakage 52 hours after NR-ERCP. Perforation of the PAD was confirmed by an emergency open surgery and repair of duodenal diverticulum was performed.

\section{Discussion}

Compared with cholangiography in conventional ERCP, DCS is advantageous by providing a real-time three dimensions direct view of the biliary tree and intraluminal lesions, thus improves the performance of NR-ERCP $[9,10,15]$.Although DCS can be used as a novel ERCP platform, DCS-assisted NR-ERCP is limited performed. This is due to not only the high cost of DCS, but also some worries about severe adverse events. Subsequently, DCS-assisted NR-ERCP should be further discussed. In this study, we presented our experience of this theme. 
Non-radiation biliary cannulation is technically feasible for experienced endoscopists. According to the study from Shah JN [7], their successful rate of non-radiation biliary access was about $85 \%$. However, successful biliary access rate can be achieved at 98\%-100\% $[9,10,16]$. In our case series, non-radiation biliary access successful rate was $98.1 \%$, and was comparable to that of fluoroscopy guided biliary cannulation. In the study by Ridtitid W [9], technical success of DCS-assisted ERCP for stone clearance was similar with that of conventional ERCP. In their study, a cholangiogram was set as the gold standard for complete stones clearance. However, as they described, DCS-based biliary exploration itself is sufficient for confirmation of stones clearance [9]. In two recent studies [10, 15], DCS-based non-radiation stone extraction was achieved in 40 and 21 patients. Taking advantage of a definite intracavitary view, DCS viewing for biliary exploration was performed instead of cholangiography. Additionally, details of stones and outlet of bile duct will contribute to determination of therapeutic strategy. As showed in Fig. 2, cracked stones were present by DCS viewing, which was different from prior imaging, and planned lithotripsy was remitted. Time length of biliary exploration was similar with the study by Ridtitid W [9]. DCS can be easily performed by an experienced and well trained endoscopist $[9,17]$. It is not technically difficult to perform DCS-based biliary exploration even in patients with a non-dilated CBD. Taken together, DC-assisted NR-ERCP is technically feasible for endoscopic extraction of CBD stones.

Application of DCS-guided lithotripsy will facilitate endoscopic retrieval of large and difficult stones [11, $16,18,19]$. However, DCS-guided lithotripsy was performed in a hybrid endoscopic and fluoroscopic procedure $[11,17,18]$, and fluoroscopy time was not shortened by using DCS-guided lithotripsy [16]. Barakat MT et al reported their experience of DCS-guided electrohydraulic lithotripsy in few unexpectedly complex cases [10]. Their study also showed that DCS-guided lithotripsy was technically feasible in a non-radiation manner. In other studies $[9,10,15]$, patients with large stones were not included in DCSassisted NR-ERCP. So there may be a bias of patient enrollment, and feasibility of non-radiation lithotripsy for large and difficult stones should be further discussed. Our performance is the first report on nonradiation DCS-guided laser lithotripsy for large stones. In our case series, about 17\% patients needed lithotripsy in DCS group, and stone clearance was achieved in all patients. Our results showed that DCSassisted ERCP is superior to mechanical lithotripsy in conventional ERCP for retrieval of large and difficult CBD stones by presenting a complete stone clearance and less ERCP sessions. Since endoscopic retrieval for difficult CBD stones clearance is a time-consuming procedure [16], both patients and endoscopy staff can be remitted from radiation induced hazard.

Some key details are listed as follows. Firstly, since fluoroscopy is not used, a prior imaging is important, and a short time interval between a prior imaging and an ERCP is recommended. Secondly, there may some worries about inadvertent cannluation into a cystic duct, bile leakage and perforation. Although there is no randomized trials available, direct visualization has the potential to address many of the questions of NR-ERCP [15]. Similar to our results, there was no report of such adverse events in the previous studies $[9,10,15]$. Also it may be doubtable if retained stones may be missed. In the hands of expert endoscopists, approximate amount of stones can be judged according to prior imaging $[9,15]$. Furthermore, the second round DCS biliary exploration after stone retrieval can confirm if there were residual stones [9]. Thirdly, since EPBD does not increase incidence of post-ERCP pancreatitis [20], small 
EST combined with EPBD may be recommended. Compared with standard EST, it can provide a relatively larger orifice of distal bile duct [21,22], thus decrease the water pressure load during cholangioscopy. Consequently, risks of post-ERCP complications, including cholangitis and hemorrhage, can be controlled

and reduced. In case of unexpectedly relatively larger or impacted stones, real-time laser lithotripsy can be used $[9,10]$.

Safety of DCS-assisted NR-ERCP should be discussed. According to the study by Lenze F et al [11], a rate of severe adverse events at $16.4 \%$ was reported. In the present study, total and severe adverse events rate were $17 \%$ and $7.6 \%$, respectively. This is similar with that of the study by Ridtitid W [9]. The most severe adverse event was a delayed type II perforation due to a type III PAD. PAD is a risk of ERCP-related complications [23, 24]; however, the incidence was about $1 \%$ by an expert [24-26]. According to the study by Ridtitid W [9], there was a $2 \%$ type II perforation caused by EST in conventional ERCP; however, there was no perforation in DCS-assisted ERCP by performing EST. According to a recent study, a delayed bile duct perforation may occur in patients with large PAD even at seventh day post papillary large balloon dilation [25]. Subsequently, it should be carefully manipulated by performing a limited EST and a smaller EPBD in presence of a large PAD. In our case series, despite of no prophylactic antibiotics administration, there was no cholangitis. Besides limited insufflations during cholangioscopy, performance of small EST combined with EPBD and prophylactic biliary stenting may also be contributed. Our results and previous data $[9,10]$ showed DCS-based NR-ERCP can be safely performed.

There are some limitations to this study. First, this is a retrospectively study by enrolling patients from different time stages. Although conventional ERCP was performed th the same time, about 3/4 patients were willing to receive NR-ERCP. Consequently, previous patients who underwent conventional ERCP were set as a control. Second, this is a single-center study. This is due to SpyGlass DS ${ }^{\mathrm{Tm}}$ is available in some academic hospitals in China; and NR-ERCP is performed by few endoscopits. Third, a cost-effectiveness analysis is not performed. However, since the cost of a low-dose X-ray system is much more expensive than that of a cholangioscopy, this strategy provides an option for hospitals which tend to update the ERCP platform.

\section{Conclusions}

Taken together, our data indicate that DCS-based NR-ERCP is technically feasible and safe for retrieval of CBD stones. This approach provides an alternative to standard ERCP.

\section{List Of Abbreviations}

DCS: digital cholangioscopy

NR-ERCP : non-radiation endoscopic retrograde cholangiopancreatography

CBD: common bile duct 
EST: endoscopic sphincterotomy

EPBD: endoscopic papillary balloon dilation

PAD: periampullary diverticulum

\section{Declarations}

This study was approved by the review board Zhongda Hospital affiliated to Southeast University (approval number: 2019ZDSYLL093-P01) and was in accordance with the Declaration of Helsinki and its later amendments. A copy of written consent was obtained from each patient before ERCP.All authors agree to publish this manuscript in BMC Surgery.

All data and materials were available in the manuscript. There was no conflict of interest. No funding was received for this study.

Yadong Feng, Wei Xu and Yang Liu performed NR-ERCP and DCS. Xiaomei Sun, Chibin Bu, Youyu Zhang and Jiongzhang were in charge of post-ERCP clinical observation. Yanliang performed statistical analysis. Yangdong Feng and Ruihua Shi designed this study. We thanked Ms. Xia Chen, Youzhen Zhang, Qingmei Ji, Danhui Zhu and Jiating Yuan for their help in NR-ERCP performance.

\section{References}

1. Manes G, Paspatis G, Aabakken L. et al. Endoscopic management of common bile duct stones: European Society of Gastrointestinal Endoscopy (ESGE) guideline. Endoscopy. 2019;51:472-91.

2. Campbell N, Sparrow K, Fortier M. et al. Practical radiation safety and protection for the endoscopist during ERCP. Gastrointest Endosc. 2002;55:552-7.

3. Liao C, Thosani N, Kothari S, Friedland S. et al. Radiation exposure to patients during ERCP is significantly higher with low-volume ednsoscopists. Gastrointest Endosc. 2015;81:391-8.

4. Garg MS, Patel P, Blackwood M. et al. Ocular radiation threshold projection based off of fluoroscopy time during ERCP. Am J Gastroenterol. 2017;112:716 - 21.

5. Muniraj T, Aslanian HR, Laine L. et al. A double-blind, randomized, sham-controlled trial of the effect of a radiation-attenuating drape on radiation exposure to endoscopy staff during ERCP. Am J Gastroenterol. 2015;110:690-6.

6. Domonceau JM, Garcia-Fernandez FJ, Verdun FR. et al. Radiation protection in digestive endoscopy: European Society of Digestive Endoscopy (ESGE) guideline. Endoscopy. 2012;44:408 - 21.

7. Shah JN, Bhat YM, Hamerski CM. et al. Feasibility of nonradiation EUS-based ERCP in patients with uncomplicated choledocholithiasis (with video). Gastrointest Endosc. 2016;84:764-9.

8. Sethi S, Thosani N, Banerjee S. Radiation-Free ERCP. in Pregnancy: A "Sound" Approach to Leaving No Stone Unturned. Dig Dis Sci. 2015;60:2604-7. 
9. Ridtitid W, Luangsukrerk T, Angsuwatcharakon P, et al. Uncomplicated common bile duct stone removal guided by cholangioscopy versus conventional endoscopic retrograde cholangiopancreatography. Surg Endosc. 2018;32:2704-12.

10. Barakat MT, Girotra M, Choudhary A, et al. A prospective evaluation of radiation-free direct solitary cholangioscopy for the management of choledocholithiasis. Gastrointest Endosc. 2018;87:584-9.

11. Lenze F, Bokemeyer A, Gross D, et al. Safety, diagnostic accuracy and therapeutic efficacy of digital single-operator cholangioscopy. United European Gastroenterol. 2018;6:902-9.

12. Kiriyama S, Kozaka K, Takada T, et al. Tokyo Guidelines 2018: diagnostic criteria and severity grading of acute cholangitis (with videos).J Hepatobiliary Pancreat Sci. 2018; 25:17-30.

13. Cotton PB, Lehman G, Vennes J, et al. Endoscopic sphincterotomy complications and their management: an attempt at consensus. Gastrointest Endosc. 1991;37:383-93.

14. Rex DK, Deenadayalu VP, Eid E, et al. Endoscopist-directed administration of propofol: a worldwide safety experience. Gastroenterology. 2009;137:1229-37.

15. Shelton J, Linder JD, Rivera-Alsina ME, et al. Commitment, confirmation, and clearance: new techniques for nonradiation ERCP during pregnancy (with videos). Gastrointest Endosc. 2008;67:364-8.

16. Park SY, Park CH, Lim SU, et al. Intraductal US-directed management of bile duct stones without radiocontrast cholangiography. Gastrointest Endosc. 2015;82:939-43.

17. Weigt J, Kandulski A, Malfertheiner P. Technical improvement using ultra-slim gastroscopes for direct peroral cholangioscopy: analysis of the initial learning phase. J Hepatobiliary Pancreat Sci. 2015;22:74-8.

18. Buxbaum J. Sahakian A, Ko C, et al. Randomized trial of cholangioscopy-guided laser lithotripsy versus conventional therapy for large bile duct stones (with videos). Gastrointest Endosc. 2018; 87: 1050-60.

19. Ogura T. Imanishi M, Kurisu Y, et al. Prospective evaluation of digital single-operator cholangioscope for diagnostic and therapeutic procedure (with videos). Dig Endosc. 2017; 29: 782-9.

20. Jang SI, Yun GW, Lee DK. Balloon dilation itself may not be a major determinant of post-endoscopic retrograde cholangiopancreatography pancreatitis. World J Gastroenterol. 2014;20:16913-24.

21. Okuno M, Iwashita T, Yoshida K, et al. Significance of endoscopic sphincterotomy preceding endoscopic papillary large balloon dilation in the management of bile duct stones. Dig Dis Sci. 2016;61:597-602.

22. Kuo CM, Chiu YC, Liang CM, et al. The efficacy of limited endoscopic sphincterotomy plus endoscopic papillary large balloon dilation for removal of large bile duct stones. BMC Gastroenterol. 2019;19:93.

23. Bray MS, Borgert AJ, Folkers ME. et al. Outcome and management of endoscopic retrograde cholangiopancreatography perforations: A community perspective. Am J Surg. 2017;214:69-73. 
24. Karaahmet $F$, Kekilli M. The presence of periampullary diverticulum increased the complications of endoscopic retrograde cholangiopancreatography. Eur J Gastroenterol Hepatol. 2018;30:1009-12.

25. Zulli C, Grande G, Tontini GE. et al. Endoscopic papillary large balloon dilation in patients with large biliary stones and periampullary diverticula: Results of a multicentric series. Dig Liver Dis. $2018 ; 50: 828-32$.

\section{Figures}
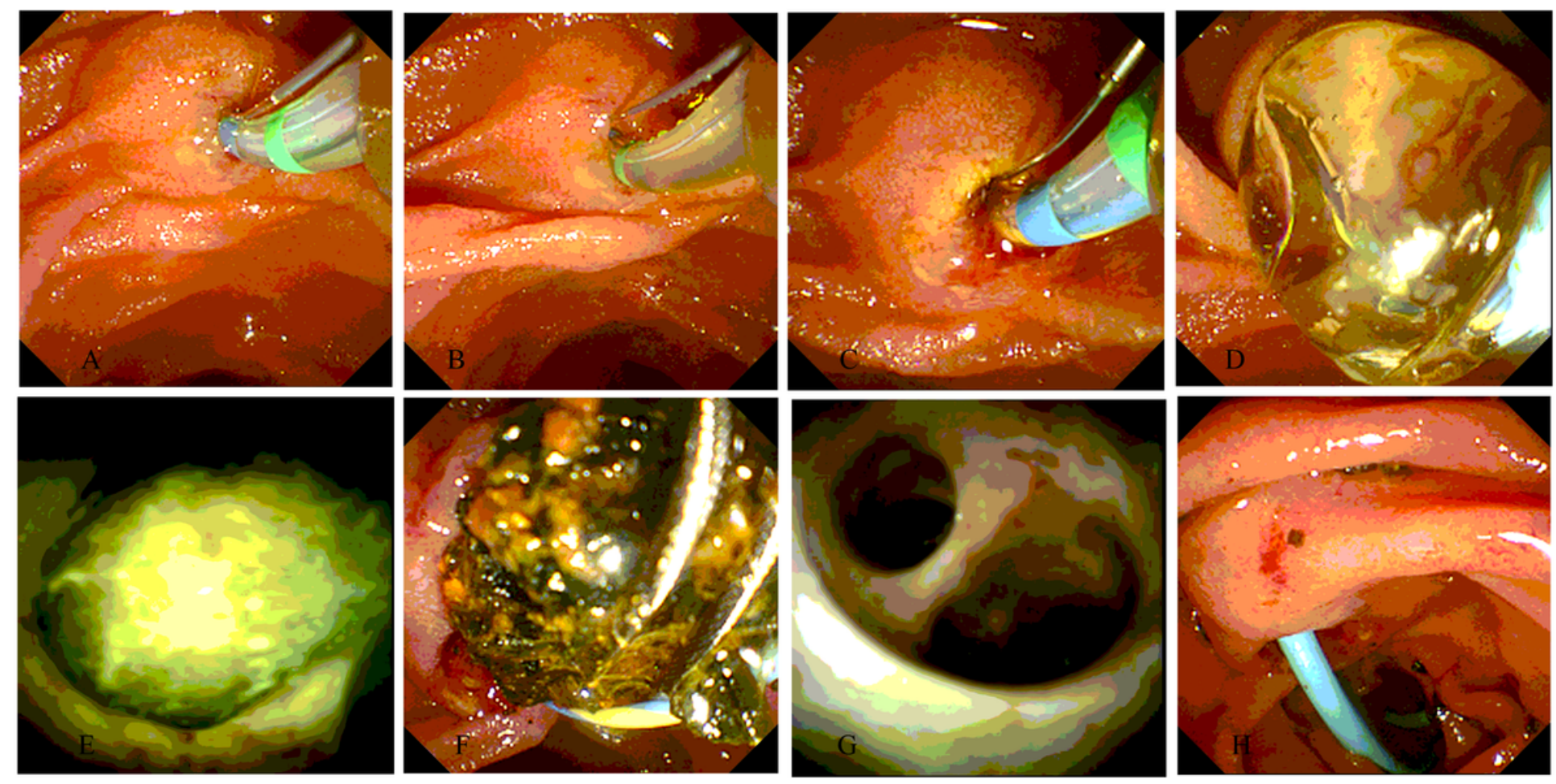

Figure 1

Key steps of NR-ERCP for retrieval of CBD stones. (A) Non-radiation biliary cannulation using a sphincterotomeand a 0.035-inch guidewire. (B)Successful biliary cannulation confirmed by visible bile aspiration in the catheter. (C) A small endoscopic sphincterotomy. (D) Endoscopic papillary balloon dilation. (E) Direct view of a CBD stone by a DCS-based non-radiation biliary exploration. (F) Basket CBD stone extraction.(G) A second round DCS to confirm CBD stone clearance. (H) A 7F double pig-tail stent placement for temporary biliary drainage. 


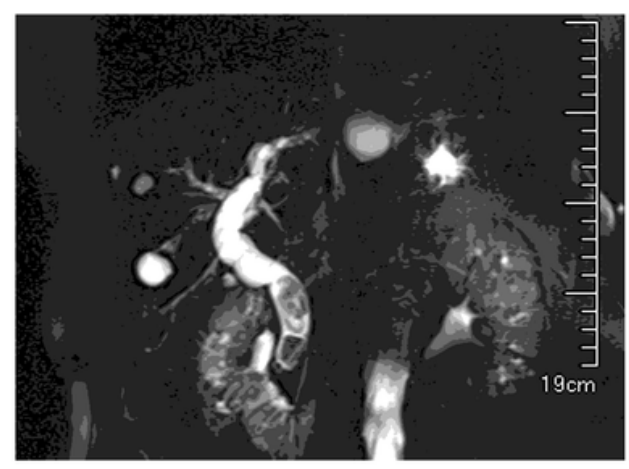

A

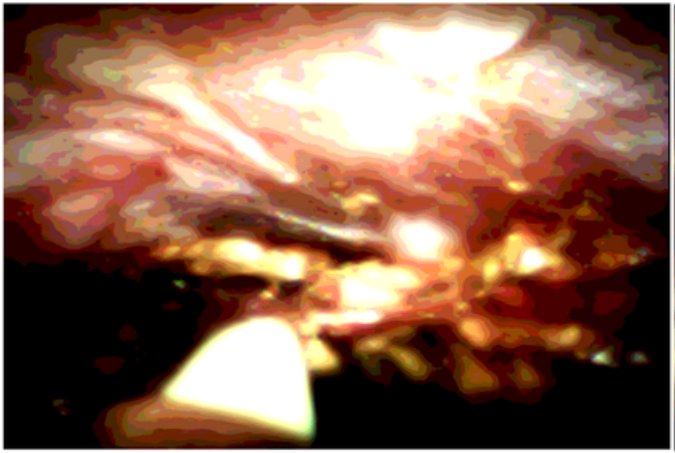

B

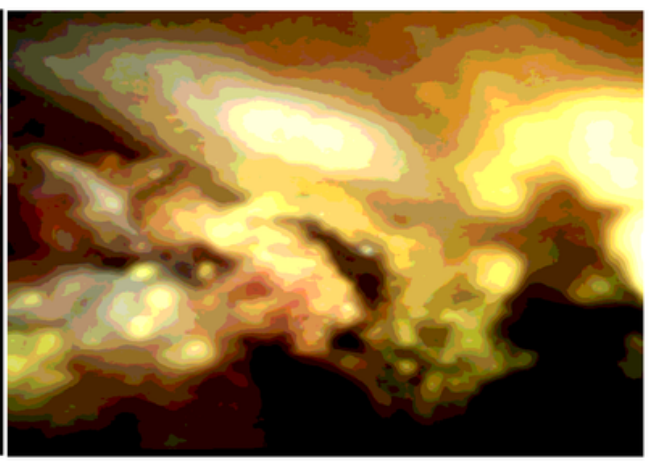

$\mathrm{C}$

Figure 2

One case of cracked stones confirmed by DCS-based biliary exploration. (A) Multiple large CBD stones presented by a piror MRI. (B) and (C) Crakced CBD stones conformance by a DCS-based biliary exploration. 